Nogo-A improves regenerative and plastic responses after spinal cord injury. Neuron $2003 ; 38$ : 201-11.

9. Steward 0 , Zheng B, Banos $K$, et al. Axon regeneration in young adult mice lacking Nogo-A/B. Neuron 38, 187-199. Neuron 2007; $54: 191-5$.

10. Zheng B, Atwal J, Ho C, et al. Genetic deletion of the Nogo receptor does not reduce neurite inhibition in vitro or promote corticospinal tract regeneration in vivo. Proc Natl Acad Sci USA 2005 ; 102 : 1205-10.

11. Lee JK, Geoffroy CG, Chan AF, et al. Assessing spinal axon regeneration and bourgeonnement in Nogo-,
MAG-, and OMgp-deficient mice. Neuron 2010 ; $66: 663-70$.

12. Lee JK, Chan AF, Luu SM, et al. Reassessment of corticospinal tract regeneration in Nogo-deficient mice. J Neurosci $2009 ; 29: 8649-54$.

13. Lee JK, Case LC, Chan AF, et al. Generation of an OMgp allelic series in mice. Genesis 2009 $47: 751-6$.

14. Li C, Tropak MB, Gerlai R, et al. Myelination in the absence of myelin-associated glycoprotein. Nature $1994 ; 369: 747-50$.
15. Cafferty WB, Strittmatter SM. The Nogo-Nogo receptor pathway limits a spectrum of adult CNS axonal growth. J Neurosci $2006 ; 26: 12242-50$

16. Cafferty WB, Duffy P, Huebner $\varepsilon$, et al. MAG and OMgp synergize with Nogo-A to restrict axonal growth and neurological recovery after spinal cord trauma. J Neurosci $2010 ; 30: 6825-37$

17. Elliott J, Cayouette M. La protéine de polarité Par-3. Chef d'orchestre dans la formation de la myéline. Med Sci (Paris) 2007 ; 23 : 259-60.

\title{
NOUVELLE
}

\section{Syndrome de Kallmann}

\section{Une séquence fœtopathologique}

Fabien Guimiot, Luis Teixeira, Catherine Dodé, Anne-Lise Delezoide, Jean-Pierre Hardelin
F. Guimiot, A.L. Delezoide : Service de fœtopathologie, Hôpital Robert Debré, 48, boulevard Sérurier, 75019 Paris, France. L. Teixeira, C. Dodé : Institut Cochin, Département de génétique et développement, 24, rue du Faubourg Saint-Jacques, 75014 Paris, France. J.P. Hardelin : Institut Pasteur, Département de neuroscience, 25, rue du Docteur Roux, 75015 Paris, France. jean-pierre.hardelin@pasteur.fr
Sexe et nez : un lien embryologique... La gonadolibérine ou gonadotropin-releasing hormone ( $\mathrm{GnRH}$ ) contrôle l'axe hormonal de la reproduction. Des études embryologiques effectuées depuis une vingtaine d'années chez les mammifères, les oiseaux et les poissons ont montré que les cellules neuroendocrines qui synthétisent cette hormone proviennent de l'épithélium nasal et migrent vers le télencéphale en empruntant le trajet des nerfs olfactifs. Chez les mammifères, elles migrent en fait le long des fibres du nerf voméro-nasal (impliqué dans la perception des phéromones) et du nervus terminalis (dont le rôle dans la reproduction reste mal connu) [1]. Parvenues à la base du télencéphale, elles y pénètrent, avec les fibres du nervus terminalis, juste en arrière de l'ébauche des bulbes olfactifs, et cheminent ensuite dans la partie interne des futurs hémisphères cérébraux jusqu'aux régions préoptique et hypothalamique, où elles établiront la neurosécrétion endocrine. Dans l'espèce humaine, la migration de ces cellules débute au cours de la $6^{\mathrm{e}}$ semaine de vie embryonnaire. C'est aussi la période où les fibres des nerfs olfactifs et celles des nervus terminalis établissent leur premier contact avec le cerveau, peu de temps avant l'émergence des bulbes olfactifs.
... et physiopathologique

Le syndrome de Kallmann associe un hypogonadisme hypogonadotrope, dû à un déficit en gonadolibérine, et une anosmie (ou hyposmie) accompagnée d'une aplasie des bulbes olfactifs [2]. Le plus souvent, le diagnostic est fait à l'âge de la puberté, devant l'absence d'apparition des caractères sexuels secondaires. L'examen histopathologique d'un fœtus de sexe masculin âgé de 19 semaines et porteur, dans la région Xp22.3, d'une grande délétion chromosomique incluant le gène KALI responsable de la forme récessive liée au chromosome $X$ de ce syndrome, avait permis, dès 1989 , de formuler l'hypothèse d'un défaut de la migration des cellules neuroendocrines à l'origine de l'hypogonadisme du syndrome de Kallmann. Chez ce fœtus, qui n'avait pas de bulbes olfactifs, les cellules neuroendocrines étaient en effet absentes du cerveau: elles s'étaient accumulées dans la région naso-frontale avec les terminaisons des nerfs olfactif et terminalis, dont les fibres interrompues n'établissaient pas de contact avec le télencéphale [3] (Figure 1B).

L'arhinencéphalie, c'est-à-dire l'absence des bulbes olfactifs, qui est l'une des anomalies du syndrome de Kallmann, est également observée dans différentes maladies pléiotropes du développement, notamment dans le syndrome CHARGE (l'appellation «maladie CHARGE» serait d'ailleurs plus appropriée), dont l'incidence est estimée à 1 sur 8500 à 12500 naissances. L'acronyme anglais CHARGE désigne l'association atrésie des choanes, cardiopathie congénitale, retard de croissance, hypoplasie des organes génitaux et anomalies de l'oreille externe et interne [4]. Cependant, de nombreux individus atteints de cette maladie n'ont pas toutes les anomalies phénotypiques qui composent l'acronyme et en ont par ailleurs souvent d'autres [5]. Une étude assez récente a conclu en particulier à la coexistence, chez la plupart des patients, d'un hypogonadisme hypogonadotrope et d'une anosmie avec arhinencéphalie, c'està-dire des deux anomalies qui définissent le syndrome de Kallmann [6]. Le syndrome CHARGE est dû, dans environ deux tiers des cas, à une mutation dans le gène CHD7 qui code une protéine de liaison à l'ADN impliquée dans la transcription de certains gènes. Des mutations de ce gène ont également été identifiées chez quelques individus pour lesquels le diagnostic initialement porté était celui d'un syndrome de Kallmann, renforçant ainsi l'idée d'une parenté nosologique 

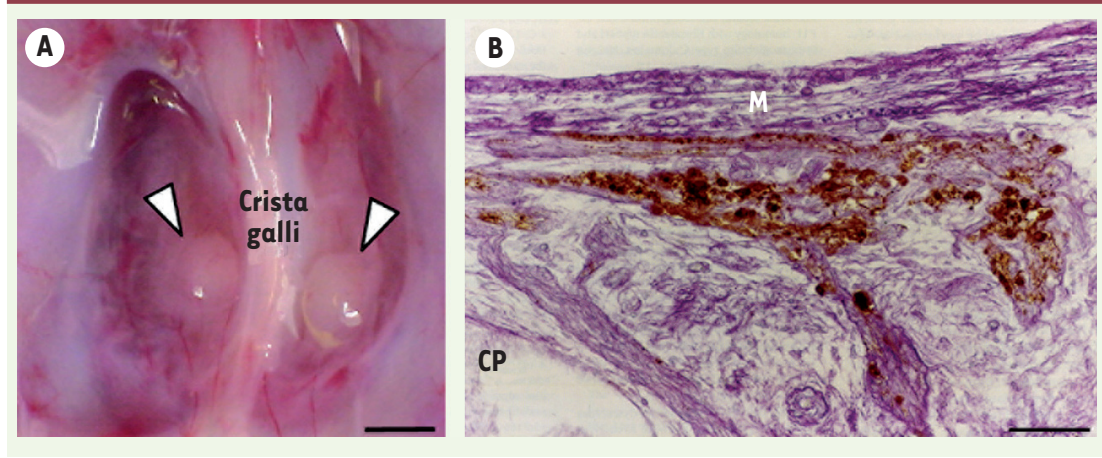

Figure 1. Pathogenèse $d u$ syndrome de Kallmann: la séquence fœtopathologique olfacto-génitale. A. Vue endocrânienne de la région de la lame criblée de l'ethmoïde chez un fœtus de 23 semaines atteint du syndrome CHARGE, maladie pléiotrope du développement incluant un syndrome de Kallmann. Les têtes de flèche indiquent, de part et d'autre de l'apophyse crista galli, des névromes d'environ $1 \mathrm{~mm}$ de diamètre, formés par l'enchevêtrement des fibres interrompues des nerfs olfactif

et terminalis. Barre d'échelle : $1 \mathrm{~mm}$. B. Coupe parasagittale de la région naso-frontale chez un fœtus KALl de 19 semaines atteint de la forme liée au chromosome $X$ du syndrome de Kallmann. Contre-coloration au violet de crésyl. Des amas de cellules synthétisant la GnRH (colorées en brun par une réaction immunochimique grâce à un anticorps spécifique de ce décapeptide), dont la migration vers le télencéphale est interrompue, sont visibles entre la face dorsale de la lame criblée de l'ethmoïde (l'abréviation CP se réfère au nom anglais, cribriform plate) et la dure-mère (M, méninge) (barre d'échelle : $100 \mu \mathrm{m}$ ).

entre ces deux entités [7, 8]. II était donc logique de se demander si l'hypogonadisme du syndrome CHARGE résultait lui aussi d'un défaut de la migration embryonnaire des cellules synthétisant la GnRH. L'arhinencéphalie se rencontre aussi dans la trisomie 13 ou la trisomie 18 [9]. Ces trisomies s'accompagnent d'ailleurs souvent, chez le garçon, d'une cryptorchidie et d'un micropénis, signes évocateurs d'un hypogonadisme hypogonadotrope. Nous avons donc étudié, par immunomarquage sur des coupes du cerveau antérieur et des coupes de la région naso-frontale, la localisation des cellules synthétisant la GnRH chez des fœtus humains arhinencéphales atteints du syndrome CHARGE, de trisomie 13, ou de trisomie 18, pour lesquels la gravité des malformations associées avait conduit à interrompre la grossesse audelà de 18 semaines de développement. Dans tous les cas, nous n'avons détecté que peu ou pas de cellules neuroendocrines dans les régions préoptique et hypothalamique où elles auraient dû se trouver à ce stade du développement. $\varepsilon$ n revanche, de nombreuses cellules synthétisant la $\mathrm{GnRH}$ étaient présentes dans la région naso-frontale de ces fœtus, reproduisant ainsi la situation déjà décrite chez le fœtus atteint du syndrome de Kallmann dans sa forme liée au chromosome $X$. Chez certains de ces fœtus, les fibres interrompues des nerfs olfactif et terminalis formaient même, par leur enchevêtrement, des névromes d'environ $1 \mathrm{~mm}$ de diamètre, visibles à la face endocrânienne de la lame criblée de l'ethmoïde et recouverts par la dure-mère [10] (Figure 1A).

Une séquence pathologique commune, des causes génétiques diverses

Ces observations, effectuées sur des fœtus atteints d'arhinencéphalie d'origines génétiques différentes, permettent de conclure à l'existence d'une nouvelle séquence fœtopathologique, que nous proposons de nommer séquence olfacto-génitale, par laquelle une anomalie précoce de développement du système olfactif périphérique entraîne un hypogonadisme par déficit hypothalamique en cellules neuroendocrines, du fait d'un arrêt de leur migration dans la région naso-frontale (Figure 2 ). Le syndrome de Kallmann (anosmie + hypogonadisme hypogonadotrope) en est la conséquence clinique. Ce syndrome, hétérogène au plan génétique, peut résulter de la mutation d'un gène unique (KAL1, FGFR1, FGF8, PROKR2, PROK2, CHD7, etc.), de plusieurs mutations synergiques dans le cadre d'une maladie digénique ou oligogénique comme cela a déjà été montré dans quelques cas, ou encore, plus rarement, s'intégrer dans un syndrome de gènes contigus en rapport avec la délétion de gènes voisins sur un chromosome (région Xp22.3 où se situe KALl, région 8p11-p12 où se situe FGFRI) [2]. Il peut aussi être associé à la duplication d'un chromosome entier comme nous venons de le montrer par l'analyse des fœtus atteints de trisomie 13 ou 18 [10]. Bien que les gènes dont la surexpression est à l'origine de la séquence olfacto-génitale ne soient pas encore identifiés sur les chromosomes 13 et 18 , et en supposant que ces gènes soient uniques, il est tentant de penser que certains individus atteints $d u$ syndrome de Kallmann pourraient être porteurs, dans ces gènes, d'une mutation entraînant un « gain de fonction ». Au plan clinique, le syndrome de Kallmann peut être isolé, ou associé à un ou plusieurs autres signes résultant d'anomalies du développement en dehors des systèmes olfactif ou reproducteur. La présence et la nature de ces signes additionnels dépendent du ou des gènes mutés [2]. Ces signes peuvent donc, lorsqu'ils sont présents, orienter la recherche de mutations chez un individu atteint du syndrome de Kallmann dans le cadre d'un conseil génétique. Ainsi, l'existence de syncinésies controlatérales d'imitation (mouvements en miroir) ou d'une agénésie rénale conduit-elle à rechercher d'abord une mutation de KALI, tandis 


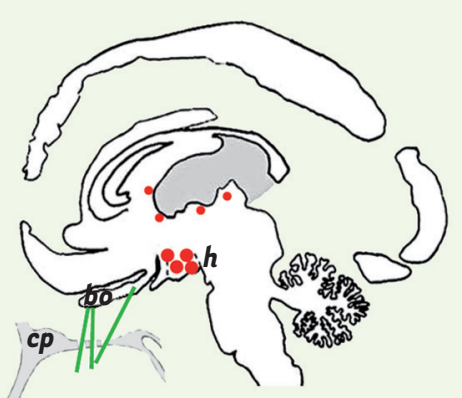

Fœtus témoin

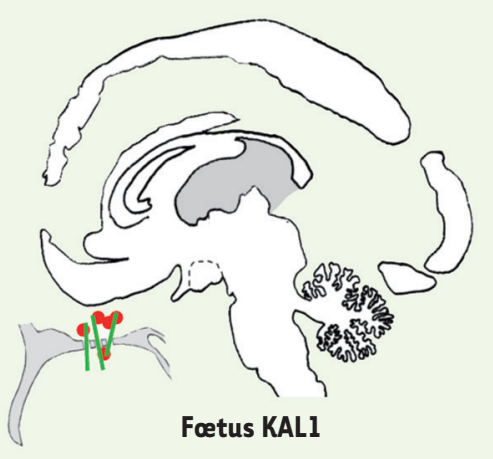

Figure 2. Distribution des cellules synthétisant la GnRH. Représentation schématique de la distribution des cellules synthétisant la $\mathrm{GnRH}$ chez un fœtus témoin de 24 semaines et chez un fœtus arhinencéphale KALl du même âge, porteur d'une mutation dans le gène responsable de la forme liée au chromosome $X$ du syndrome de Kallmann. Les traits verts indiquent le trajet des fibres des nerfs olfactif et terminalis. Les points rouges représentent l'emplacement des cellules synthétisant la $\mathrm{GnRH}$. Chez le fœtus témoin, la plupart de ces

cellules sont localisées dans l'aire préoptique et la région hypothalamique ( $h$ ). Quelques cellules éparses, vraisemblablement égarées au cours de leur migration, sont détectées à proximité du ventricule latéral (coloré en gris). Chez le fœetus KALl, la migration des cellules synthétisant la GnRH s'est arrêtée avant leur pénétration dans le télencéphale. Elles se sont accumulées dans la région naso-frontale, avec les fibres interrompues des nerfs olfactif et terminalis qui n'ont pas non plus établi de contact avec le cerveau. Noter l'absence des bulbes olfactifs (bo) chez ce fœtus. Cp : lame criblée de l'ethmoïde (cribriform plate).

que la présence d'une fente labiale et/ ou palatine fait rechercher d'abord une mutation de FGFRI ou FGF8. Certaines anomalies comme l'hypodontie (absence d'une ou plusieurs dents) ou l'hypoacousie (baisse de l'acuité auditive) peuvent, quant à elles, résulter de la mutation de différents gènes. Quant au syndrome CHARGE, véritable maladie pléiotrope $d u$ développement, sa variabilité phénotypique a été envisagée précédemment, mais l'agénésie des canaux semi-circulaires de l'oreille interne (à rechercher par l'imagerie tomodensitométrique des rochers) semble y être quasi constante [5].

Moins d'un tiers des individus atteints du syndrome de Kallmann (en dehors du syndrome (HARGE) sont porteurs d'une mutation dans un des gènes déjà identifiés, ce qui indique que d'autres gènes impliqués dans ce syndrome restent à découvrir. Leur identification permettra notamment de déterminer, à côté des cas de transmission authentiquement monogénique du syndrome, la proportion des cas de transmission digénique ou oligogénique qui demeure inconnue à ce jour. $\diamond$

Kallmann syndrome, a fetopathological sequence

\section{CONFLIT D'INTÉRÊTS}

Les auteurs déclarent n'avoir aucun conflit d'intérêts concernant les données publiées dans cet article.

\section{RéFÉRENCES}

1. Schwanzel-Fukuda M, Pfaff DW. Origin of luteinizing hormone-releasing hormone neurons. Nature 1989 ; 338: 161-4.

2. Dodé C, Hardelin JP. Kallmann syndrome. Eur J Hum Genet $2009 ; 17: 139-46$.

3. Schwanzel-Fukuda M, Bick D, Pfaff DW. Luteinizing hormone-releasing hormone (LHRH)-expressing cells do not migrate normally in an inherited hypogonadal (Kallmann) syndrome. Mol Brain Res 1989; 6 : 311-26.

4. Pagon RA, Graham JM Jr, Zonana J, Yong SL. Coloboma, congenital heart disease, and choanal atresia with multiple anomalies: CHARGE association. J Pediatr $1981 ; 99: 223-7$.

5. Sanlaville D, Verloes A. CHARGE syndrome: an update. EurJ Hum Genet 2007 ; 15 : 389-99.

6. Pinto G, Abadie V, Mesnage R, et al. CHARGE syndrome includes hypogonadotropic hypogonadism and abnormal olfactory bulb development. J Clin Endocrinol Metab 2005 ; 90 : 5621-6.

7. Kim HG, Kurth I, Lan F, et al. Mutations in CHD7, encoding a chromatin-remodeling protein, cause idiopathic hypogonadotropic hypogonadism and Kallmann syndrome. Am J Hum Genet 2008; $83: 511-9$.

8. Jongmans MC, van Ravenswaaij-Arts CM, Pitteloud N, et al. CHD7 mutations in patients initially diagnosed with Kallmann syndrome: the clinical overlap with CHARGE syndrome. Clin Genet 2009 ; 75 : 65-71.

9. Delezoide AL, Narcy F, Larroche JC. Cerebral midline developmental anomalies: spectrum and associated features. Genet Couns 1991; $1: 197-210$.

10. Teixeira L, Guimiot F, Dodé C, et al. Defective migration of neuroendocrine $\mathrm{GnRH}$ cells in human arrhinencephalic conditions. J Clin Invest 2010 ; $120: 3668-72$.

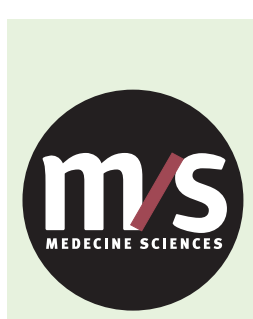

Tarifs d'abonnement M/S - 2011
Abonnez-vous
à Médecine/Sciences

$>$ Grâce à $m / s$, vivez en direct les progrès des sciences biologiques et médicales

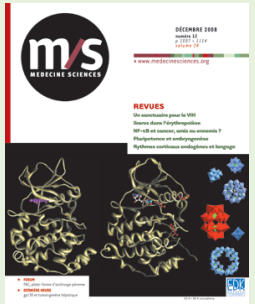
page 152 dans ce numéro de $\mathrm{m} / \mathrm{s}$
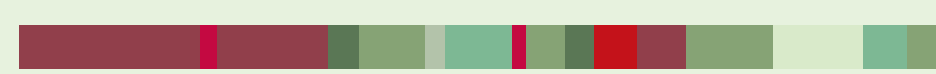